\title{
Clinician's Perceptions and Expectations on a mHealth Platform for Supporting Patient Data Integration and Clinical Service Delivery: A Case Study in Evidence-Based Communication Rehabilitation
}

\author{
Erhhsuan Wang \\ Health Information Management \\ University of Pittsburgh \\ erw39@pitt.edu
}

Valerie Watzlaf

Health Information Management

University of Pittsburgh

valgeo@pitt.edu

\author{
Leming Zhou \\ Health Information Management \\ University of Pittsburgh \\ Leming.Zhou@pitt.edu
}

\author{
Bambang Parmanto \\ Health Information Management \\ University of Pittsburgh \\ parmanto@pitt.edu
}

\author{
Mervat Abdelhak \\ Health Information Management \\ University of Pittsburgh \\ abdelhak@pitt.edu
}

\begin{abstract}
Improving the quality of healthcare services while simultaneously reducing the overall cost remains a huge challenge. One recommended approach for achieving this goal is to build high quality data collection and reporting systems to facilitate evidencebased practice (EBP), which emphasizes the importance of using the solid evidence available to make optimal clinical decisions. In this project, we conducted interviews and a survey with clinicians to understand their perceptions and expectations on such data collection and reporting systems. Based on the obtained results, we created an integrated platform using mobile and web technologies for Augmentative and Alternative Communication (AAC) intervention. A usability study was conducted to evaluate the integrated platform and compared the platform to other existing data collection and reporting approaches. The study results indicated that our platform provides a better approach for supporting data collection, integration, and reporting, as well as streamlining the workflow of AAC clinical service delivery.
\end{abstract}

\section{Introduction}

A report released in 2013 by the Institute of Medicine (IOM) highlighted the significant demand for improving healthcare quality and reducing its cost in the United States [1]. One of the recommended approaches is to create high-quality data collection and reporting systems, since such systems can provide real- time access to patient data, translate the collected data into medical evidence, and as a result, facilitate evidence-based practice (EBP), which emphasizes the importance of using the best evidence available to make optimal clinical decisions. For that purpose, clinicians are required to collect, analyze, report, and review their patients' performance data before they adjust treatment.

Verbal communication is an essential skill to human beings and a major influence on the overall quality of life. However, over 3.7 million people in the U.S. have a profound communication disability -- they cannot express themselves with their voice [2, 3]. Augmentative and Alternative Communication (AAC) intervention, including assistive technology and strategies, has been proven to be effective in enabling people with communication disabilities (PwCD) to express themselves, increase social interaction, and maintain independent communication [4].

For AAC clinical service delivery, EBP requires speech language pathologists (SLPs) to collect and review clients' language performance data and use the best evidence available to make clinical decisions [4]. Some traditional AAC devices can be used to collect user activity data and generate log files; however, the reports cannot be generated in the same treatment session without extensive manual work, and the log files cannot be retrieved remotely [5]. In recent years, A few mobile AAC applications (apps) have become available to support automatic outcome report generation in real time. Unfortunately, those reports provide limited information for monitoring clinical outcomes. Therefore, the lack of efficient clinicallyrelevant data collection and reporting in AAC 
intervention profoundly affects the process of clinical service delivery.

Mobile technologies have been widely adopted throughout healthcare service delivery in recent years [6]. The portability and accessibility of mobile devices present an obvious opportunity for supporting a better quality of care in the domain of healthcare. Mobile devices may be used for a wide variety of purposes, such as disease diagnosis and assessment [7, 8], disease management and monitoring [9, 10], disease prevention $[11,12]$, health education or promotion [13, $14]$, and data collection and reporting $[15,16]$. These studies suggested that there are potential benefits in using mobile devices to provide more efficient, accessible, lower cost, and better quality of healthcare services. The advantages of mobile apps for real-time data collection and retrieval are very useful for healthcare professionals in patient monitoring [17]. A web portal may serve as a resource for providing healthcare professionals with convenient access to comprehensive information about their patients' clinical performance.

The adoption of mobile technologies in healthcare services has provided new ways of capturing user activity data and providing real-time outcomes. Patient-generated data can be used to monitor patient outcomes, improve treatment strategies, and translate information into medical evidence by providing realtime access to knowledge. Integrating mobile devices and a web-based portal could serve as a platform to provide EBP in AAC service delivery.

\section{Background}

\subsection{Evidence-based Practice}

EBP is the integration of best research evidence with clinical expertise and patient data [18]. It emphasizes the importance of using the most solid evidence available to make optimal clinical decisions. In the field of communication science, EBP is defined as the conscientious, explicit, and judicious integration of 1) best available external evidence from systematic research; 2) the best available evidence internal to clinical practice; and 3) the best available evidence concerning the preferences of a fully informed patient [4, 19]. High-quality external evidence from systematic research can reveal valuable information about average patterns of performance across groups of patients, however, its applicability to an individual patient is unknown [20]. Internal evidence to clinical practice with a patient, in other words, objective patient data, are important complements to external evidence from systematic research. Internal evidence and the patient's preference can be collected through quantitative analysis of language samples [4]. To realize EBP in communication science, one needs to make this type of patient data readily available to SLPs.

\subsection{AAC - Augmentative and Alternative Communication}

According to American Speech-Language-Hearing Association, "People with severe speech or language problems rely on AAC to supplement existing speech or replace speech that is not functional [21].” AAC refers to any communication approaches that supplement or replace natural speech and/or writing that may be impaired [4]. It includes devices, various types of content used to express needs and thoughts, and clinical strategies to enhance or replace natural speaking abilities. AAC can be considered as an approach of communication, as well as a tool to develop language skills. AAC strategies can range from no-technology (paper and pencil) to hightechnology (electronic devices with vocal output). The AAC technique has been proven to enable people with communication disabilities to express themselves, increase social interaction, and maintain independent communication across the lifespan [4].

\subsection{Existing Data Collection and Reporting Approaches in AAC}

2.3.1. Approach 1: Collecting Data Using Dedicated AAC Device and Manually Generating Reports. In this approach, language samples such as the content and time of language events are collected and stored in dedicated AAC devices. The language samples are stored as text files in these devices. To obtain these files, SLPs need to physically obtain the devices and retrieve the text file through a USB flash drive when their clients are visiting the clinic. To generate a report, SLPs need to manually preprocess the collected language samples and feed the processed text file into a special computer program to generate a performance report. This is a delayed and time-consuming process. SLPs can only retrieve the data when their clients are visiting the clinic and SLPs also need to spend a lot of time to preprocess the collected data before they can obtain the final performance report. Therefore, when this approach is used, SLPs do not know their clients' most recent performance in communication when they see their clients in the clinic. Because this report generation process is manual and time-consuming, and the information is always delayed, a number of SLPs choose not to download and process the language samples collected by the dedicated AAC devices [22]. 
2.3.2. Approach 2: Collecting Data and Automatically Creating Reports Using Mobile AAC Apps. The second data collection and reporting approach was implemented in mobile AAC apps. These types of apps are able to collect clients' data and generate performance reports in real-time. The timestamp of each event is also included in the report. SLPs can either review the result directly in the app or ask their clients (or caregivers of their clients) to email the report to them through the app. This approach is more convenient than the previous approach, however, SLPs still can only access the reports when the clients are visiting their clinic (view on the app) or specifically request the clients to email the reports to them. To make the situation even more cumbersome, the data items in those reports are often not exactly what SLPs are most interested in because the designers of those AAC apps are not researchers and therefore, did not seek the input from SLPs when they created the apps [22].

\subsection{Theoretical Framework}

The overall theoretical framework of this project is the Systems Development Life Cycle (SDLC) [23], which is highly recommended for producing highquality software systems that meet customer's expectations. The typical stages of SDLC include a) requirement analysis, b) software system design and implementation, c) system evaluation, and d) system deployment and maintenance. Although these software development stages are well known to the health IT community, many mobile health (mHealth) systems were created and released to the market without the analysis of user requirements and therefore many of these mHealth systems did not meet the needs of target users [24]. This paper provides a description of the first three stages of our mHealth platform development and evaluation, with extensive details on the first stage: requirement analysis. The second and third stages are described briefly in this article and further details are provided in other places [22, 25].

\section{Methods}

To create a data collection, integration, and reporting system that will be highly useful for SLPs, the first step is to perform requirement analysis. In other words, we need to know the perceptions and expectations SLPs have on the specific data items and information they desire to see in the data collection, integration, and reporting system. For that purpose, we conducted an interview and a survey study with SLPs.

\subsection{Interview Study}

SLPs in the greater Pittsburgh area were recruited through professional referrals. Each interview took roughly one hour, and all interviews were audio recorded for further data analysis. The following are the questions used in the interview study. They are organized into six different categories.

\section{a) Background questions}

- How many years of work experience do you have as a SLP?

- What is your current work setting?

- Have you provided AAC intervention to your clients?

\section{b) Data collection approaches}

- Do you collect data (e.g. language samples) during the AAC intervention? If yes, please describe your data collection methods.

- What is your main purpose for data collection?

- Are you able to monitor clients' everyday activities on AAC devices/apps in real time? If yes, please describe your approach.

- Are you satisfied with your current data collection approach? Why or why not?

- What are the problems/limitations of the current data approach? Are there any changes that you expect to see in the near future?

c) Perspectives on mobile AAC apps

- Have you used any mobile AAC apps before? Do you like those apps? Why or why not?

- What are the advantages and disadvantages of mobile AAC apps in your opinion?

\section{d) AAC therapy outcome measurements}

- Which outcome measurements do you want to see in collected data?

- Are there any specific outcome measurements you use for your reports for evaluation and reimbursement purposes?

\section{e) Desired features on a web-based clinician portal}

- Do you think the following features of a webbased clinician portal are beneficial for you? Why or why not?

- Monitoring clients' performance anytime through the Internet

- Providing a summary of clients' language performance over time

- Providing outcome details for further analysis

- Are there any other features that you would expect to see? 
f) Perspective on a web-based clinician portal

- Do you believe such a web portal will support AAC service delivery?

- Will the clinician portal enhance the current data collection and reporting process?

- Will the clinician portal improve both the quality and efficiency of health service delivery?

\subsection{Survey Study}

The interview study was used to obtain the general idea about the current data collection approaches and SLPs' desire for a better data collection and reporting system, in addition to the general requirements on the outcome data. This survey study was to understand SLPs' opinion on performance data and the specific types of information that SLPs consider important to clinical intervention and outcome measurements. For that purpose, an online survey was conducted with SLPs via the Qualtrics system in the United States (Qualtrics, Provo, Utah). This survey was an updated version of a validated questionnaire named AAC Sampling Procedures and Performance Monitoring Questionnaire (SPPMQ) published in 2001 [5]. This new version of the survey covered questions about SLPs' perspectives on traditional language sampling methods, as well as their expectations to recent innovative data collection and analysis procedures. Some questions were updated because of advancement in information technology in recent years, such as the wide adoption of mobile apps in healthcare. Table 1 shows some updates to survey questions and the corresponding reasons for the specific update. The recruitment flyers of this survey study were distributed during the annual Clinical AAC Research Conference in 2015 and via the AAC Institute Newsletter.

\subsection{System Implementation}

A language sample data collection, integration, and reporting platform (named EuTalk) was then created based on a comprehensive literature review, the information collected in the interview and survey studies using mobile and web technologies.

\subsection{System Evaluation}

A usability study on EuTalk was conducted with communication science professionals to evaluate its usability of the system. EuTalk was also compared with other existing data collection and reporting approaches. Professionals from the field of communication science between the ages of 18 and 65 were recruited for this study, including certified SLPs and graduate students with clinical experience. The study protocol was approved by the Institutional Review Board (IRB) at the University of Pittsburgh.

Table 1. Updates made on SPPMQ and the corresponding reasons.

\begin{tabular}{|c|c|c|}
\hline SPPMQ & $\begin{array}{l}\text { New } \\
\text { Questionnaire }\end{array}$ & Reasons for Update \\
\hline & $\begin{array}{l}\text { List mobile AAC } \\
\text { apps that are } \\
\text { presently used. }\end{array}$ & $\begin{array}{l}\text { Added a new } \\
\text { question regarding } \\
\text { the use of mobile } \\
\text { AAC apps }\end{array}$ \\
\hline $\begin{array}{l}\text { Use videotape } \\
\text { to collect } \\
\text { language } \\
\text { samples. }\end{array}$ & $\begin{array}{l}\text { Use video } \\
\text { recording to } \\
\text { collect language } \\
\text { samples. }\end{array}$ & $\begin{array}{l}\text { Videotape is rarely } \\
\text { used recently. Video } \\
\text { recording is more } \\
\text { common today }\end{array}$ \\
\hline $\begin{array}{l}\text { Indicate the } \\
\text { use of the } \\
\text { methods to } \\
\text { collect AAC } \\
\text { performance } \\
\text { data. }\end{array}$ & $\begin{array}{l}\text { Indicate the use } \\
\text { of the automatic } \\
\text { methods to } \\
\text { collect language } \\
\text { samples. }\end{array}$ & $\begin{array}{l}\text { The question was } \\
\text { updated to ask about } \\
\text { automatic data } \\
\text { collection methods } \\
\text { since most of the } \\
\text { manual data } \\
\text { collection methods } \\
\text { are no longer used }\end{array}$ \\
\hline $\begin{array}{l}\text { Indicate the } \\
\text { selection of } \\
\text { word-based } \\
\text { and } \\
\text { utterance- } \\
\text { based } \\
\text { summary } \\
\text { measures. }\end{array}$ & $\begin{array}{l}\text { Indicate the } \\
\text { selection of } \\
\text { utterance-based, } \\
\text { word-based, and } \\
\text { app-based } \\
\text { summary } \\
\text { measures. }\end{array}$ & $\begin{array}{l}\text { App-based summary } \\
\text { measures were } \\
\text { added to include } \\
\text { app-specific data } \\
\text { analysis }\end{array}$ \\
\hline $\begin{array}{l}\text { SLPs' } \\
\text { perspectives } \\
\text { on the data } \\
\text { logging } \\
\text { feature on the } \\
\text { AAC device. }\end{array}$ & $\begin{array}{l}\text { SLPs' } \\
\text { perspectives on } \\
\text { the data logging } \\
\text { feature on the } \\
\text { mobile AAC app. }\end{array}$ & $\begin{array}{l}\text { To understand } \\
\text { SLPs' perspectives } \\
\text { on the mobile AAC } \\
\text { apps }\end{array}$ \\
\hline
\end{tabular}

During the usability study, the study participants were introduced to EuTalk and two other data collection and reporting approaches in AAC technologies described in section 2.3. These study participants were required to finish several tasks (such as managing a client list, generating and reviewing performance reports, and managing client records) using each data collection and reporting approach. Upon completion of all assigned tasks, the participants were asked to complete a post-study questionnaire to express their overall impression on EuTalk. The study participants were also asked to compare these three data collection and reporting approaches and indicate their preferences in different scenarios. 


\section{Results}

In this section, the results from the interview study, the survey study, and a brief summary of the system evaluation are provided. Extensive details of the system evaluation studies go beyond the scope of this article and are described in [22, 25] and one manuscript currently in preparation.

\subsection{Results of Interview Study}

\section{a) Background}

Five certified SLPs in the greater Pittsburgh area were recruited to participate in this interview study. Participants' work experiences ranged between 4 to 12 years. Most of them (80\%) were SLPs with more than 5 years of work experience. Participants' current work settings included private clinics and schools. All participants had experiences in AAC intervention.

\section{b) Existing Approaches of Data Collection}

The existing approaches for data collection include behavior observation (taking notes), video/audio recording, and downloading text files from dedicated AAC devices. Behavior observation is the method that most participants used daily. Reimbursement and treatment adjustment are two main purposes for which SLPs would collect clients' performance data. All participants agreed that they are not satisfied with the current data collection approach, since it takes extra time to collect and process the data. Moreover, participants are not able to monitor clients' everyday activities in real time. To improve the current data collection approach, the participants expect to have a new approach that is able to automatically collect real time data and provide quality information.

\section{c) Perspective on Mobile AAC Apps}

Four of the five (80\%) participants had experiences using mobile AAC apps. They all agreed that mobile AAC apps have the potential to help people with communication disabilities. However, the quality of the existing AAC apps is one of their biggest concerns, because most mobile AAC apps on the market were not designed by healthcare professionals and did not collect desired data for clinical purposes.

\section{d) Outcome Measurements}

Four of the five (80\%) participants indicated that the client's time of using AAC devices is important to their communication performance. Other outcome measurements include communication rate, mean length of utterance, and error rate. Three (60\%) participants expressed a need to distinguish the logs by different users, such as clients vs. family members.

\section{e) Perspective on a Web-based Clinician Portal}

All participants believed that a clinician portal could support AAC service delivery, enhance the current data collection and reporting process, and ultimately improve both the quality and efficiency of health service delivery.

\section{f) Desired Features on a Web-Based Portal}

All participants agreed that some features on the clinician portal could be beneficial, such as remote monitoring in real time and a dashboard overview, as well as detailed outcome information. They suggested including the ability to select the time period of the data and various options for exporting data.

\subsection{Results of Survey Study}

Twenty-six responses were collected in this online survey study. The work experience the participants had in providing AAC clinical services ranged from 1 year through 34 years (mean $=12$ years). Participants collected language samples using handwritten, audio recordings, or video recordings approaches. Fifty-four percent of the participants collected language samples from dedicated AAC devices, while $44 \%$ of them collected data from mobile AAC apps.

Participants answer other survey questions in this study on a 4-point Likert scale: agree, somewhat agree, somewhat disagree, and disagree.

Ninety-five percent of the participants agreed that analyzed performance data would be helpful for providing more effective therapy and $66 \%$ of the participants agreed that the information would make it possible for producing faster progress in the AAC intervention process.

Ninety-five percent of the participants agreed the analyzed data would benefit people who rely on AAC, $90 \%$ agreed that it would better clarify needs, and $81 \%$ agreed that it would lead to more effective communication. Furthermore, $90 \%$ of the participants agreed that the performance data provides more effective data to justify the selection of an AAC system. Sixty-two percent of the participants agreed that a mobile device for data collection is easy and practical.

Seventy-two percent of the participants agreed that the use of built-in data logging feature of an AAC app is easy and practical, and $90 \%$ percent of the participants would be more likely to use this built-in feature. Ninety percent of the participants agreed that the availability of automated performance monitoring tools will allow for more frequent collection of 
language samples, and 95\% agreed that using such a tool can improve the analysis process. Consequently, 95\% of the participants agreed that these tools should be easy and user-friendly to integrate the tool into the intervention program. Moreover, 95\% of the participants would collect performance data if the performance monitoring tools were able to make the process easy and efficient, while 90\% would use analyzed performance data more often if the tools were available as an entire package.

The outcome measurements most respondents believed important ( $\geq 70 \%$ ) include three app-based, three word-based, and four utterance-based summary measurements. More specifically, they are usage frequency of the app, user language performance at home, percentage of core vocabulary, total number of words, utterance structure, total utterances, average communication rate in words, mean length of utterances in words, usage frequency of different activities in the app, and total number of different words.

\subsection{Implemented System: EuTalk}

A mobile AAC app and a web-based clinician portal (EuTalk) were implemented according to the findings identified in the interview and the survey studies, in addition to a comprehensive literature review. More specifically, the comprehensive literature review identified problems in the current AAC systems and existing data collection and reporting approaches, while the interview and survey studies indicated the desired data items, specific language performance measurements, and SLPs desired features of the new mHealth platform.

The tablet-based AAC app was developed to provide a communication interface for everyday communication and clinical treatment exercises for PwCD [25]. The app logs all user activities and transmits the data to a secure server over the Internet and the data on the server are only accessible to authorized SLPs via the web portal.

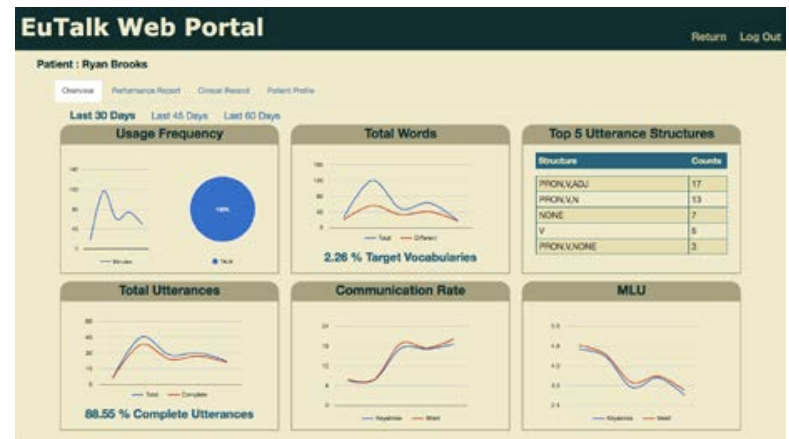

Figure 1. A web-based clinician portal - Dashboard.
The web portal was developed to provide an overview and detailed information about PwCD's communication performance to SLPs. The dashboard (Figure 1) provides SLPs an easy-to-read interface to examine clients' progress reports in graphical charts. SLPs can also access the raw performance data and detailed analysis results while PwCD are using the mobile app at home. With this information on the portal, clinicians can obtain a better idea about their clients' communication routines and overall progress over time.

The complete data collection provides clinicians with the best available evidence to improve clinical decision-making. Clinicians can adjust their treatment approaches and personalize app settings for each client accordingly with a few clicks on the web portal.

\subsection{System Evaluation Results}

4.4.1. Study Participants. Ten participants were recruited to participate in this system evaluation study, including five certified SLPs in the greater Pittsburgh area and five graduate students with clinical experience in the Department of Communication Science and Disorders at the University of Pittsburgh. All 10 participants completed the assigned tasks and provided feedback through questionnaires $(\mathrm{N}=10)$. None of these participants attended the interview study described earlier. Participants had ages between 22 and 61 (mean $=28.4, \mathrm{SD}=11.71)$. Eighty percent of participants were female and $20 \%$ were male. The length of time that each participant has provided AAC clinical services ranged from half a year through 30 years $($ mean $=4.4, \mathrm{SD}=8.65)$. Here $\mathrm{SD}$ means standard deviation. The entire study took each participant roughly an hour to complete, including finishing all assigned tasks and filling out usability questionnaires. The time each study participant spent on each assigned task was fairly brief (seconds to minutes) since all they needed to do was to create sentences in the new mobile AAC app and view the results in the web portal via a few button clicks. The entire procedure went smoothly and no participant had difficulties in finishing those assigned tasks.

4.4.2. Overall Usability of EuTalk. The Post Study System Usability Questionnaire (PSSUQ) was adopted to measure the usability of EuTalk [26]. All ten participants completed the usability questionnaire after they finished the assigned tasks on EuTalk. Participants responded to the statements in PSSUQ on a 5-point Likert scale, from 1 (strongly disagree) to 5 (strongly agree). In other words, higher numbers indicated greater satisfaction with the system. The overall average rate in the usability study was 4.18 out 
of 5, which indicated that the study participants believed the usability of EuTalk was high.

4.4.3. SLP's Preferences on Data Collection and Reporting Systems. The participants were asked to compare EuTalk with two existing data collection and reporting approaches described in section 2.3. Ninety percent of the participants selected EuTalk as the preferred approach for supporting clinical intervention, while $10 \%$ selected the dedicated AAC device for that purpose. Eighty percent of participants chose EuTalk as the preferred data collection and reporting approach while $10 \%$ participants chose mobile app and $10 \%$ participants chose the dedicated AAC device. Furthermore, 90\% of the participants selected EuTalk as the approach that they would recommend their organizations to adopt, while $10 \%$ selected the dedicated AAC device. The results show that the participants agreed that EuTalk could better support data collection and reporting in AAC service delivery.

\section{Discussion}

In this project, a research-based approach was used to design, implement, and evaluate the integrated mHealth platform for AAC clinical service delivery. The mobile app provides PwCD an opportunity to use the app as an AAC intervention tool and deliver the clinically relevant data to SLPs without any extra efforts of PwCD, their caregivers, or SLPs. The webbased portal provides SLPs with an effective supporting tool for data analysis, integration, and report generation.

Since the rehabilitation process is usually long and frustrating, the performance reports can not only help SLPs to design appropriate treatment plans but also help PwCD to gain confidence, which fulfills the desired outcome of EBP. Our mHealth platform may therefore improve user engagement, as well as help SLPs to adjust the treatment plans according to solid patient data, support their clinical evaluation, and ultimately streamline the workflow (reduce costs) and improve the quality of their clinical service.

There are some limitations in this project. First, only one usability study of the system was conducted. Patients' clinical performance after the adoption of the entire system in AAC clinics was not evaluated yet. Second, the usability study focused on the viability of the system, and the SLPs' preference on the newly implemented mHealth system and two existing approaches. The quantitative study on user satisfaction with each approach was not conducted. Although the overall result showed the integrated platform offers a better analytics approach, the statistical difference was not determined. In our future work, we will design clinical trials to collect patients' clinical performance data and monitor SLPs' engagement in this system.

\section{Conclusion}

In this project, a mixed method, including an interview and a survey with SLPs, was used to understand SLPs' perceptions and expectations on data collection and reporting in AAC therapy. An integrated mHealth platform (EuTalk) was created according to the interview and survey study results. The usability study on EuTalk and a brief survey on SLPs' preferences on data collection and reporting approaches indicated that SLPs were satisfied with EuTalk and preferred to use EuTalk in their clinical practice. In other words, the integrated mHealth platform offers a better clinical data collection and reporting approach for AAC clinical services. Also, it may facilitate evidence based communication rehabilitation service delivery, which may make it possible to improve the quality of the healthcare services and reduce the cost at the same time.

\section{Acknowledgments}

This research is funded in part by grants from the National Institute on Disability, Independent Living, and Rehabilitation Research (NIDILRR grant numbers 90RE5018, 90DP0064, and 90DPGE0002). NIDILRR is a Center within the Administration for Community Living (ACL), Department of Health and Human Services (HHS). We would like to thank Dr. Katya Hill for giving us the permission to use and modify the validated SPPMQ questionnaire in this study.

\section{References}

[1] Institute of Medicine, "Best care at lower cost: The path to continuously learning health care in America," Washington, DC, 2013.

[2] D. Beukelman and P. Mirenda, "Augmentative and alternative communication: Supporting children and adults with complex communication needs," 4th edition: Paul H. Brookes Publishing Company, Baltimore, MD, 2013.

[3] M. W. Brault, "Americans with disabilities: 2010," U. S. Census Bureau, 2012.

[4] K. Hill, B. Baker, and B. A. Romich, "Augmentative and Alternative Communication technology," in An Introduction to Rehabilitation Engineering, R. A. Cooper, H. Ohnabe, and D. A. Hobson, Eds., Taylor \& Francis, 2006.

[5] K. Hill, "The development of a model for automated performance measurement and the establishment of performance indices for augmented communicators under 
two sampling conditions," PhD Dissertation, University of Pittsburgh, Pittsburgh, PA, 2001.

[6] M. Fiordelli, N. Diviani, and P. J. Schulz, "Mapping mHealth research: A decade of evolution," Journal of Medical Internet Research, vol. 15, p. e95, 2013.

[7] S. Kumar, E.-H. Wang, M. J. Pokabla, and R. J. Noecker, "Teleophthalmology assessment of diabetic retinopathy fundus images: smartphone versus standard office computer workstation," Telemedicine and e-Health, vol. 18, pp. 15862, 2012.

[8] H. Takao, Y. Murayama, T. Ishibashi, K. L. Karagiozov, and T. Abe, "A new support system using a mobile device (smartphone) for diagnostic image display and treatment of stroke," Stroke, vol. 43, pp. 236-9, 2012.

[9] A. G. Logan, W. J. McIsaac, A. T. Tisler, M. J. Irvine, A. Saunders, A. Dunai, et al., "Mobile phone-based remote patient monitoring system for management of hypertension in diabetic patients," American Journal of Hypertension, vol. 20, pp. 942-8, 2007.

[10] E. Mattila, J. Parkka, M. Hermersdorf, J. Kaasinen, J. Vainio, K. Samposalo, et al., "Mobile diary for wellness management-Results on usage and usability in two user studies," IEEE Transactions on Information Technology in Biomedicine, vol. 12, pp. 501-12, 2008.

[11] H. Cole-Lewis and T. Kershaw, "Text messaging as a tool for behavior change in disease prevention and management," Epidemiologic Reviews, vol. 32, pp. 56-69, 2010.

[12] R. Whittaker, S. Merry, E. Dorey, and R. Maddison, "A development and evaluation process for mHealth interventions: Examples from New Zealand," Journal of Health Communication, vol. 17 Suppl 1, pp. 11-21, 2012.

[13] B. S. Gerber, M. R. Stolley, A. L. Thompson, L. K. Sharp, and M. L. Fitzgibbon, "Mobile phone text messaging to promote healthy behaviors and weight loss maintenance: A feasibility study," Health Informatics Journal, vol. 15, pp. 17-25, 2009.

[14] E. T. Jordan, E. M. Ray, P. Johnson, and W. D. Evans, "Text 4 Baby," Nursing for Women's Health, vol. 15, pp. 206-12, 2011.

[15] M. Tomlinson, W. Solomon, Y. Singh, T. Doherty, M. Chopra, P. Ijumba, et al., "The use of mobile phones as a data collection tool: A report from a household survey in South Africa," BMC Medical Informatics and Decision Making, vol. 9, p. 51, 2009.
[16] P. Yu, M. de Courten, E. Pan, G. Galea, and J. Pryor, "The development and evaluation of a PDA-based method for public health surveillance data collection in developing countries," International Journal of Medical Informatics, vol. 78, pp. 532-42, 2009.

[17] C. L. Ventola, "Mobile devices and apps for health care professionals: Uses and benefits," Pharmacy and Therapeutics, vol. 39, p. 356-64, 2014.

[18] D. L. Sackett, W. S. Richardson, W. Rosenberg, and R. B. Haynes, "Evidence-based medicine: How to practice and teach EBM," Second Edition. Churchill Livingstone: Edinburgh, 2000.

[19] C. A. Dollaghan, "The handbook for evidence-based practice in communication disorders," Paul H. Brookes Publishing Company: Baltimore, MD, 2007.

[20] A. C. Bohart, "Evidence-based psychotherapy means evidence-informed, not evidence-driven," Journal of Contemporary Psychotherapy, vol. 35, pp. 39-53, 2005.

[21] American Speech - Language - Hearing Association. Augmentative and Alternative Communication (AAC). URL: http://www.asha.org/public/speech/disorders/AAC/

[22] E. H. Wang, "A mHealth platform for supporting clinical data integration and service delivery: An example from Augmentative and Alteranative Communication intervention," PhD Dissertation, University of Pittsburgh, Pittsburgh, PA, 2016.

[23] ISO, IEC, and IEEE, "Systems and software engineering -- Software Life Cycle Processes," in ISO/IEC/IEEE 15288, 2015.

[24] H. Zhang, H. Zhang, X. Wang, Z. Yang, and Y. Zhao, "Analysis of requirements for developing an mHealth-based health management platform," JMIR mHealth uHealth, vol. 5, p. e117, 2017.

[25] E. H. Wang, L. Zhou, S. H. Chen, K. Hill, and B. Parmanto, "Development and evaluation of a mobile AAC: A virtual therapist and speech assistant for people with communication disabilities," Disability and Rehabilitation: Assistive Technology, in press, 2017.

[26] J. R. Lewis, "IBM computer usability satisfaction questionnaires: psychometric evaluation and instructions for use," International Journal of Human-Computer Interaction, vol. 7, pp. 57-78, 1995. 